\title{
Cannabis Use Associated with Capsular Warning Syndrome and Ischemic Stroke
}

\author{
Moogeh Baharnoori, Charles D. Kassardjian, Gustavo Saposnik
}

Can J Neurol Sci. 2014; 41: 272-273

Marijuana is the most commonly consumed recreational drug among young individuals. It is generally perceived as a safe substance with minimal adverse effects. There are a few reported cases of acute cerebral events related to cannabis. The association is presumed based on the temporal relation between cannabis use and the onset of neurological symptoms ${ }^{1}$. Multifocal intracranial vasospasm is one plausible mechanism of cannabis-associated stroke. Radiological evidence of vasoconstriction suggests dynamic changes in vessel structure may lead to reduced cerebral blood flow and subsequent ischemia ${ }^{2}$. The majority of reported ischemic cerebrovascular events related to cannabis use are in the vertebrobasilar circulation or middle and anterior cerebral artery territories ${ }^{3,4}$. We present a case of sub-cortical ischemic stroke shortly after cannabis use in a young healthy individual with no identified vascular risk factors or family history of stroke.

\section{Case Report}

A 22-year-old right handed male developed left face, arm and leg weakness and slurred speech 90 minutes after smoking marijuana. At admission, his blood pressure was elevated (157/70, pulse rate: 91, sinus rhythm). Computed tomogram/CT angiogram (CT/CTA) revealed subtle hypodensity in the right lentiform nucleus with no evidence of thrombus, vasospasm or dissection. Computed tomogram perfusion showed reduced mean transit time with no associated changes in blood flow or volume (Figure A,B). He had recurrence of similar symptoms twice. His neurological deficits fully recovered in between. His vital signs were stable. Repeat CT/CTA did not show any interval change. After the fourth episode his deficits did not resolve. Intracardiac thrombus was suspected because the patient was young (without traditional stroke risk factors) and had no vessel abnormalities (such as vasospasm, stenosis, dissection, or visible clots) on the CT angiogram. Heparin was started after recurrent episodes of focal neurological deficits while waiting for an echocardiogram. Brain magnetic resonance imaging (MRI) 12 hours later showed diffusion restriction involving posterior lentiform nucleus and right corona radiate (Figure C,D). Transthoracic and transesophageal echocardiolography, hypercoagulable and autoimmune work up were unremarkable. Heparin was discontinued when echocardiograms were normal. Conventional cerebral angiogram was declined by the patient. He started smoking marijuana at the age of 15 (on average three to four joints weekly). He denied poly-substance use or concomitant alcohol consumption. Clinical assessment two months later showed left facial droop, spastic tone and residual

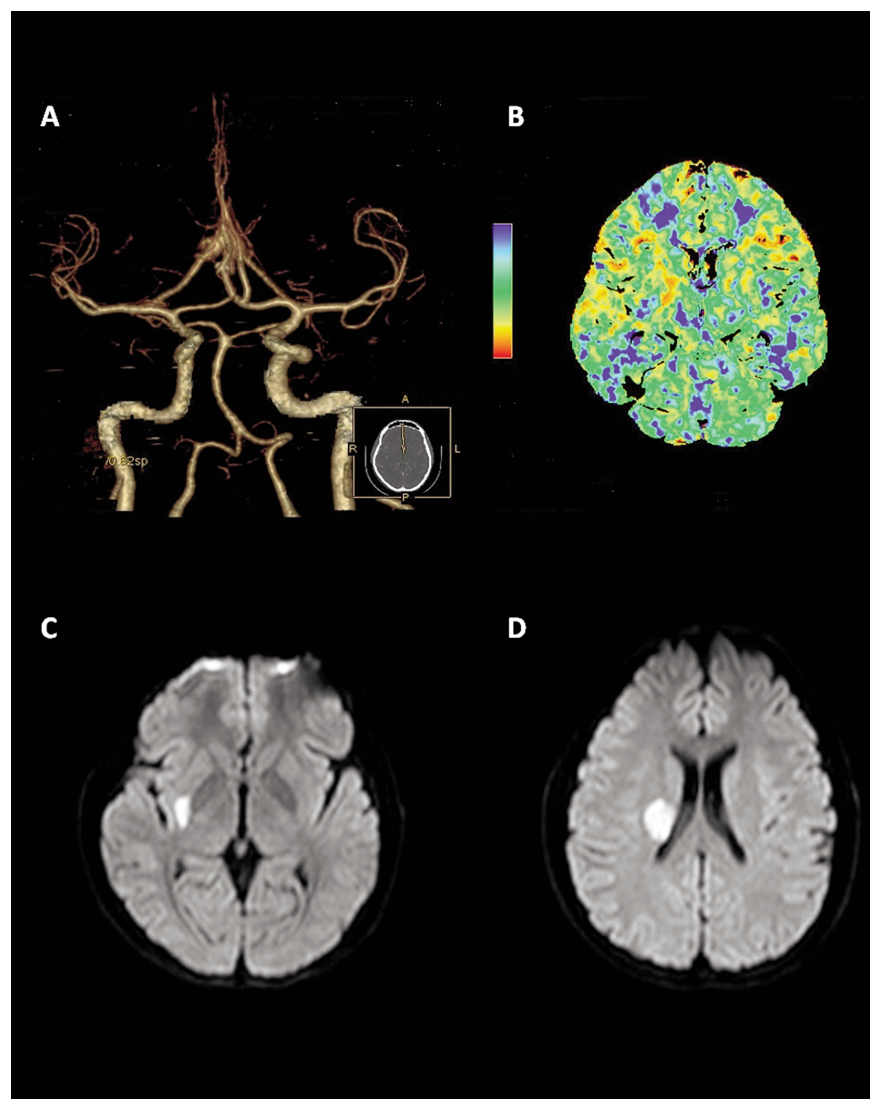

Figure: (A) CT angiogram reveals no abrupt calibre change or intraluminal filling defect in the intracranial anterior and posterior circulation. (B) Perfusion map shows reduced mean transit time (MTT) in right posterior lentiform nucleus with no corresponding changes in the blood volume. $(C, D)$ Brain MRI shows areas of restriction in diffusion weighted images (DWI) in posterior part of right lentiform nucleus and right corona radiata.

From the Division of Neurology, Department of Medicine, St Michael's Hospital, University of Toronto, Toronto, Ontario, Canada.

Received August 12, 2013. Final Revisions Submitted September 23, 2013. Correspondence to: Gustavo Saposnik, Stroke Outcomes Research Centre, Department of Medicine (Neurology), St. Michael's Hospital, University of Toronto

55 Queen St E, Toronto, Ontario, M5C 1R6, Canada.Email: saposnikg@smh.ca. 
weakness in the left arm and leg. He is no longer using cannabis and he has not developed any new neurological events. Hypercoagulable blood panel was repeated in six months and revealed no abnormalities.

\section{Discussion}

Several hypotheses have been proposed as underlying mechanisms of cannabis-related ischemic stroke. Vascular imaging in the acute phase has shown distinct patterns such as multifocal intracranial vasoconstriction and vasospasm ${ }^{2,4}$. We did not identify vasospasm in any large intracranial vessel by CT angiogram. It is possible that vasospasm affecting the collateral circulation (not usually on the CTA) rather than the main arteries, would explain the final infarct volume in the terminal territory of the MCA. This arterial territory is rarely affected based on the published cases of cannabis related stroke. In a case series by Singh et al ischemic stroke occurred in the posterior circulation in $58 \%$ of cases and in the anterior circulation in $42 \%$ of cases (mainly middle cerebral artery territories leading to cortical infarction $)^{3}$. There have been very few cases of cannabis associated infarction in subcortical regions and none of the previously published reports presented as recurrent ischemic events (capsular warning syndrome), as in our case.

Thromboembolism of cardiac origin is another potential mechanism of cannabis-related stroke. Cardiac arrhythmias such as atrial fibrillation and flutter are reported shortly after cannabis use, which may serve as a thromboembolic source. Thromboembolism was investigated as a possible mechanism for stroke in our case; continuous heart monitoring did not reveal any cardiac arrhythmias and echocardiography was negative. Systemic hypotension and vasculitis are other proposed mechanisms. Animal studies have shown that cannabis affects sympathetic tone and systemic blood pressure in a dosedependent manner. While low doses induce an increase in sympathetic activity, higher cannabinoid exposure inhibits sympathetic tone resulting in hypotension ${ }^{5}$. Impaired cerebral autoregulation is reported in experienced marijuana users ${ }^{6}$. Decreased mean transit time in our patient was attributed to luxury perfusion. Another potential explanation is an increase in cerebral perfusion due to the impaired cerebral autoregulation.

Capsular warning syndrome (CWS) is a rare phenomenon defined as recurrent stereotyped episodes of focal neurological deficits preceding a lacunar infarction. It is believed that CWS results from hemodynamic instability and impaired circulation in penetrating arteries ${ }^{7}$. Our case had ischemic stroke in the deep watershed zone, which typically occurs with hemodynamic instability. It is possible that cannabis exposure initially caused hypotension, followed by intracranial vasospasm and impaired circulation in the deep penetrating arteries. The elevated systolic blood pressure at admission might have been a secondary reflex to existing intracranial vasospasm as reported by others ${ }^{8}$. Establishing a cause and effect relationship between stroke and cannabis poses some distinct challenges. Considering the widespread use of cannabis, clinicians should be aware of the risk of ischemic stroke due to the exposure to cannabis, particularly in young adults, when all appropriate investigations are negative.

\section{Disclosures}

Dr. Gustavo Saposnik is supported by the Distinguished Clinician Scientist Award by Heart and Stroke Foundation of Canada (HSFC) following peer-review competition.

\section{REFERENCES}

1. Barber PA, Pridmore HM, Krishnamurthy V, et al. Cannabis, ischemic stroke, and transient ischemic attack: a case-control study. Stroke. 2013;44(8):2327-9.

2. Wolff V, Lauer V, Rouyer O, et al. Cannabis use, ischemic stroke, and multifocal intracranial vasoconstriction: a prospective study in 48 consecutive young patients. Stroke. 2011;42(6):1778-80.

3. Singh NN, Pan Y, Muengtaweeponsa S, Geller TJ, Cruz-Flores S. Cannabis-related stroke: case series and review of literature. J Stroke Cerebrovasc Dis. 2011;21(7):555-60.

4. Wolff V, Armspach JP, Lauer V, et al. Cannabis-related stroke: myth or reality? Stroke. 2013;44(2):558-63.

5. Adams MD, Earnhardt JT, Dewey WL, Harris LS. Vasoconstrictor actions of delta8- and delta9-tetrahydrocannabinol in the rat. J Pharmacol Exp Ther. 1976;196(3):649-56.

6. Mathew RJ, Wilson WH. Acute changes in cerebral blood flow after smoking marijuana. Life Sci. 1993;52(8):757-67.

7. Lalive PH, Mayor I, Sztajzel R. The role of blood pressure in lacunar strokes preceded by TIAs. Cerebrovasc Dis. 2003;16(1): 88-90.

8. Zachariah SB. Stroke after heavy marijuana smoking. Stroke. 1991; 22(3):406-9. 